\title{
Field-Induced SMM and Visible/NIR-Luminescence Behaviour of Dinuclear LnIII Complexes with 2-Fluorobenzoate
}

[a] Departament de Química Inorgànica i Orgànica, Secció de Química Inorgànica, Universitat de Barcelona, Martí i Franquès 1-11, 08028 Barcelona, Spain

http://www.ub.edu/inorgani/recerca/MagMol/magmol.htm

[b] Departament de Mineralogia, Cristallografia i Dipòsits Minerals and Unitat de Difracció de R-X, Centre Científic i Tecnològic de la Universitat de Barcelona (CCiTUB), Universitat de Barcelona, Solé i Sabarís 1-3, 08028 Barcelona, Spain

Ramon Vicente: rvicente@ub.edu 


\section{ABSTRACT:}

36

37 The reaction of $\mathrm{Ln}(\mathrm{NO} 3) 2 \cdot 6 \mathrm{H} 2 \mathrm{O}$ salts $(\mathrm{Ln}=\mathrm{Nd}, \mathrm{Eu}, \mathrm{Gd}, \mathrm{Tb}, \mathrm{Dy}, \mathrm{Er}$ and $\mathrm{Yb})$ with 2-fluorobenzoic acid

38 (H-2-FBz) and 1,10-phenanthroline (phen) in ethanol/water mixture allows the isolation of dinuclear

39 compounds of the formula [Ln2(2-FBz)4-(NO3)2(phen)2] $\{\mathrm{Ln}=\mathrm{Nd}(1), \mathrm{Eu}(2), \mathrm{Gd}(3), \mathrm{Tb}$ (4), Dy (5),

$40 \operatorname{Er}(6)\}$ and [Yb2(2-FBz)6(phen)2] (7). The solid-state photoluminescence study of the complexes

41 shows the $4 \mathrm{f}-4 \mathrm{f}$ lanthanide transitions in the visible range, in the cases of 2, 4 and 5 , and in the NIR

42 range for 1, 6 and 7. Magnetic studies reveal field-induced single-molecule-magnet (SMM) behaviour

43 for compounds $1,5,6$ and 7.

44 
Multifunctional molecular materials can be defined as compounds that involve coexistence, interplay or synergy between multiple physical properties.[1,2] Restricting the discussion to the combination of luminescence and single-molecule magnet (SMM) properties, the LnIII coordination compounds are ideal candidates for the construction of this kind of hybrid molecular material.[3-8] On the one hand, the $4 \mathrm{f}-4 \mathrm{f}$ electronic transitions (responsible for the light emission) are narrow and characteristic for each LnIII, and the emitting excited states are long-lived. Since the $\mathrm{f}-\mathrm{f}$ transitions are parity-forbidden, free LnIII ions have low extinction coefficients, leading to low luminescence intensity. To overcome this problem, the presence of light-harvesting ligands coordinated to the LnIII can enhance the metal luminescence through an energy transfer process, commonly known as the antenna effect.[9] The Rbenzoate ligands have been widely used in Ln coordination compounds due to the strong interaction between the LnIII ions and the oxygen atoms from the carboxylate group and also because of the strong absorbing chromophore group of the organic fragment.[10] The use of a fluorinated benzoate, such as 2fluorobenzoic acid, could enhance the NIR emission of $\mathrm{Nd}$, Er and $\mathrm{Yb}$, since the $\mathrm{C}-\mathrm{F}$ vibrational quenching is much lower than the one produced by the $\mathrm{C}-\mathrm{H}$ bonds.[11] Moreover, chelating ligands such as 1,10-phenanthroline (phen) can block two coordination sites per LnIII ion and can terminate further aggregation or potential polymerization.[12] The 1,10-phenanthroline ligand can also sensitize the luminescence of lanthanide ions through their large pi system.[13]

On the other hand, some compounds containing LnIII ions with high anisotropic magnetic moments show SMM properties.[14] Among the lanthanide ions, DyIII has yielded the largest number of 4f-based SMMs, followed by TbIII.[9,15,16,17] Nevertheless, ErIII and YbIII are also good candidates to present SMM properties.[18-20] Slow relaxation of the magnetization has been observed on several lanthanide compounds containing different $\mathrm{R}-$ benzoate ligands.[21-25] In this work, we present the structure of six new dinuclear $4 \mathrm{f}-$ metal complexes by simultaneously using bidentate bridging carboxylate groups derived from 2-fluorobenzoic acid (H-2-FBz), chelating 1,10phenanthroline (phen) and nitrato capping ligands. Four of the new reported compounds have the formula [Ln2(2-FBz)4(NO3)2(phen)2] $\{\mathrm{Ln}=\mathrm{Nd}(1), \mathrm{Eu}(2), \mathrm{Ln}=\mathrm{Gd}(3), \mathrm{Ln}=\mathrm{Dy}(5), \mathrm{Ln}=\mathrm{Er}(6)\}$ and are isostructural to the previously reported[26] [Tb2(2-FBz)4(NO3)2(phen)2] (4) compound. The coordination number of the lanthanide ions is 9 for 1-6. The same synthetic procedure for $\mathrm{Ln}=\mathrm{Yb}$ yielded a new dinuclear compound, without the nitrato ligand, with formula [Yb2(2-FBz)6 (phen)2] (7). The coordination number of the YbIII ion in this compound is 8. For the new compounds, we report here the syntheses, crystal structure, magnetic behaviour and luminescence properties. For the previously published [Tb2(2-FBz)4-(NO3)2(phen)2] (4) compound, as only the structure was reported, we have also studied its magnetic and luminescence properties. 


\section{X-ray Diffraction Crystal Structures}

Complexes 1-6 are isomorphs and crystallize in the triclinic space group $\mathrm{P}^{-}$; thus, as an example, only the structure of 2 will be discussed in detail.

\section{[Eu2(2-FBz)4(NO3)2(phen)2] (2)}

A partially labelled plot of the structure of the dinuclear compound 2 is shown in Figure 1a. Selected bond lengths are listed in Table 1. The structure of compound 2 consists of centrosymmetric dinuclear molecules in which each EuIII is nine-coordinate. The two EuIII atoms are bridged through four 2fluorobenzoate ligands with two different kinds of bridging modes. One of them is a symmetrical synsyn bridge ( $\eta 1: \eta 1: \mu$ or 2.11 using Harris notation) (Scheme 1a), with the Eu1-O1 and Eu1-O2' bond lengths being 2.3698(1) and 2.3531(1) A, respectively. The second type of 2-fluorobenzoate bridging ligands are best described as chelating-bridging ( $\eta 1: \eta 2: \mu$ or 2.21) (Scheme 1b), in which O3 acts as a bridge between the two Eu atoms, with a distance of 2.6989(1) for Eu1-O3 and 2.3705(1) Å for Eu1O3'; meanwhile, O4 is bonded only to one Eu with a 2.4066(1) Å bond length. The intramolecular Eu1 ' 'Eu1' distance is 3.9605(1) $\AA$. The coordination sphere of the metals is completed by the N1 and $\mathrm{N} 2$ atoms of a phenanthroline ligand with Eu1-N1 and Eu1-N2 bond lengths of 2.5811(1) and 2.6170(1) $\AA$, respectively, and by the O5 and O6 atoms from a nitrato anion with Eu1-O5 and Eu1-O6 bond lengths of 2.5329(1) and 2.4604(1) A, respectively. The calculation of the degree of distortion of the EuN2O7 coordination polyhedron for 2 with respect to the ideal nine-vertex polyhedron, by using continuous shape measurement theory and SHAPE software[27] shows that the EuN2O7 arrangement for 2 is intermediate between various coordination polyhedra (Table S1 in the Supporting Information). The best SHAPE calculations lead to Muffin (MFF-9), spherical capped square antiprism (CSAPR-9) and tricapped trigonal prism (JTCTPR-9) geometries, with continuous shape measure (CShM) values of $2.170,2.327$ and 2.819, respectively. The calculations of the degree of distortion of the $\mathrm{LnN} 2 \mathrm{O} 7$ coordination polyhedra for $1,3,5$ and 6 , with respect to the ideal nine-vertex polyhedron, are also shown in Table S1. A graphical representation of the metal coordination geometry of 2 is shown in Figure $1 b$.

The dinuclear entities are assembled into 1D chains in the [101] direction through $\pi$-stacking interactions between the central rings of two adjacent phenanthroline ligands from two different units (Figure 2). These chains are connected, forming a 2D sheet in the (111) plane through another $\pi$ stacking bond between two adjacent phenyl rings from the 2-F-benzoate chelating/bridging ligand. No classical hydrogen bonds are found in the crystal structures of complexes $1-3,5$ and 6 , but there is an intramolecular hydrogen bond formed by $\mathrm{C} 26-\mathrm{H} 26 \cdots \mathrm{F} 1$, with a distance of 3.115(3) $\AA$ and an angle of $136^{\circ}$ (Figure 2). The $\pi$-stacking intermolecular interaction distances and intramolecular hydrogen bond lengths and angles for compounds 1-3, 5 and 6 are summarized in Tables S2 and S3. 
120 Complex 7 also crystallizes in the triclinic space group $\mathrm{P}^{-}$and consists of a centrosymmetric dinuclear unit, but in this case, each YbIII centre presents an octacoordinate environment. A partially labelled structure of the dinuclear compound 7 is shown in Figure 3a. Selected bond lengths are listed in Table 1. The two equivalent YbIII ions are connected through four oxygen atoms from two 2-F-benzoate bridging ligands in a syn-syn coordination mode (Scheme 1a), with an intramolecular Yb1 ' ${ }^{\prime} \mathrm{Yb}^{\prime}$ distance of $5.152 \AA$. The $\mathrm{Yb}-\mathrm{O} 1$ and $\mathrm{Yb} 1-\mathrm{O} 2$ ' distances are 2.262(2) and 2.206(2) $\AA$, respectively. Each YbIII ion is bonded to two chelating 2-F-benzoates (Scheme 1c), with the Yb1-O3, Yb1-O4, Yb1-O5 and $\mathrm{Yb1-O6}$ distances ranging from 2.339 to $2.409 \AA$. The two nitrogen atoms of a phenanthroline ligand complete the coordination sphere of each ion, featuring $\mathrm{Yb} 1-\mathrm{N} 1$ and $\mathrm{Yb} 1-\mathrm{N} 2$ bond lengths of 2.459(3) and 2.385(6) A, respectively. The calculation of the degree of distortion of the YbN2O6 coordination polyhedron for 7 , with respect to the ideal eight-vertex polyhedron, by using the continuous shape measure theory and SHAPE software,[20] shows that the YbN2O6 arrangement for 7 is intermediate between various coordination polyhedra. The best SHAPE calculations lead to biaugmented trigonal prism (BTPR-8), triangular dodecahedron (TDD-8) and Johnson biaugmented trigonal prism (JBTPR-8) geometries, with CShM values of 2.551, 3.135 and 3.145, respectively. A graphical representation of the metal coordination geometry for 7 is shown in Figure $3 b$. In complex 7, there are no classical hydrogen bonds. Nevertheless, there is a weak intermolecular interaction between $\mathrm{C} 32-\mathrm{H} 32$ with a F1 atom of an adjacent dinuclear fragment that leads to the arrangement of these molecules into a 1D chain along the [011] direction, which is enhanced by a $\pi$ stacking interaction between two phenanthroline ligands, as is depicted in Figure 4. Moreover, the chains are connected, giving a 2D sheet in the (111) plane through the contact between C12-H12 and F3 from another unit (Figure 4). The intermolecular $\pi$-stacking interaction distance and intra- and intermolecular hydrogen-bond lengths and angles are summarized in Tables S2 and S3.

\section{Synthesis}

To avoid the highly energetic and not very controllable hydroor solvothermal processes, in this work, we used a straightforward room-temperature synthetic procedure, different from that used to prepare the already published complex [Tb2 (2-FBz)4(NO3)2(phen)2],[26] and have successfully obtained the latter and six new dinuclear lanthanide compounds. Five of the mentioned complexes are isostructural with the TbIII one, but when using YbIII as the lanthanide source, the complex obtained is different and has the formula [Yb2(2-FBz)6(phen)2].

It is worth noting, then, the viability of the room-temperature approach for obtaining coordination 
This family of [Ln2(2-FBz)4(NO3)2(phen)2] complexes provides an opportunity to study the influence of the lanthanide contraction over the structural arrangement. When the atomic number of the metal increases, the radius of the LnIII cation decreases, and slight changes in the coordination sphere of the Ln can be observed, as has been demonstrated in other papers.[28-30] The most important change observed herein is the two different types of structures obtained in this work by using the same synthetic approach. For NdIII, EuIII, GdIII, TbIII, DyIII and ErIII ions, a homo-dinuclear structure was obtained, in which the nonacoordinated metals are bridged by four carboxylate ligands, two in syn-syn coordination mode and two in chelating/bridging mode. Meanwhile, in the case of the dinuclear complex with the YbIII ion, with the smallest radius of the family, the coordination decreases to eight, and therefore, the two metals inside the molecule are bridged by only two carboxylate ligands in a synsyn coordination mode. Additionally, there is a terminal carboxylate, instead of the nitrato anion, present in the former structural type.

Other systematic structural variations coming from the different size of the ionic radius can be extracted for each type of structure (Table 1). Within the isostructural complexes 1-3, 5, 6 and the previously published compound 4, the bond lengths from the coordination sphere of the LnIII decrease $2-3 \%$ from Eu to Er, except for the largest Ln1-O3 bond, which presents a tendency to increase nearly $2 \%$ with the diminution of the LnIII radius. Then, for almost all of the $\mathrm{Ln}-\mathrm{O}$ and $\mathrm{Ln}-\mathrm{N}$ bonds, the lengths decrease due to the increase of the lanthanide contraction along the period. On the other hand, the intramolecular LnIII $\cdots$ LnIII distances are almost constant in the entire studied series for 1-6, with an average value of $3.960 \AA$. Due to the different coordination mode of the $2-\mathrm{FBz}$ bridging ligands in compound 7, the YbIII $\cdots$ YbIII distance is $5.152 \AA$, and it provides the largest $\mathrm{Ln}-\mathrm{Ln}$ distance between the metal atoms of all of the compounds presented in this work.

\section{Magnetic Properties}

\section{De Magnetic Susceptibility Study}

Solid-state direct-current (dc) magnetic susceptibility ( $\mathrm{M}$ ) data on polycrystalline powder samples of complexes 1-7 were collected under applied magnetic fields of $0.3 \mathrm{~T}(300-2 \mathrm{~K})$ for 1 and 3-7 and at 0.5 $\mathrm{T}(300-2 \mathrm{~K})$ for 2 . The data are plotted as $\mathrm{M} \times \mathrm{T}$ versus $\mathrm{T}$ in Figure 5. compounds. By analogy with the dn-dn dinuclear compounds, the exchange coupling interaction can be 
But the spin Hamiltonian can only be used for GdIII, since it has no orbital contribution, and therefore, no spin-orbit coupling effect.[31] The other lanthanides(III) need much more complex models, based on explicit ligand field spin-orbit parameters.[32] Usually, such treatments are not carried out, due to their complexity, so the magnetic behaviour of $1-7$ will be described, but the coupling constant $\mathrm{J}$ will only be calculated for the GdIII compound 3 .

At room temperature, the $\mathrm{M} \times \mathrm{T}$ values for compounds $1-7$ are 3.14, 2.91, 16.13, 23.88, 25.95, 23.46 and $4.93 \mathrm{~cm} 3 \mathrm{~mol}-1 \mathrm{~K}$, respectively. These data are in good agreement with the expected values for the corresponding two noninteracting LnIII-LnIII centres with ground states 4I9/2 [NdIII], 7F0 [EuIII], 8S7/2 [GdIII], 7F6 [TbIII],6H15/2 [DyIII], 4I15/2[ErIII] and 2F7/2 [YbIII].[9] Upon cooling, the $\mathrm{M} \times$ T values gradually decrease for 1,2 and 7, which should be mainly attributed to the depopulation of their excited states. At $2.0 \mathrm{~K}$, the $\mathrm{M} \times \mathrm{T}$ value are $1.17,0.03$ and $2.18 \mathrm{~cm} 3 \mathrm{~mol}-1 \mathrm{~K}$ for 1,2 and 7 , respectively, indicating an $\mathrm{mJ}=0$ ground substate for EuIII ion (7F0).

For 3 and 4, the $\mathrm{M} \times \mathrm{T}$ product remains almost constant down to ca. $30 \mathrm{~K}$ for $\mathrm{Gd}$ and ca. $45 \mathrm{~K}$ for $\mathrm{Tb}$ and then decreases to $13.41 \mathrm{~cm} 3 \mathrm{~mol}-1 \mathrm{~K}$ (for 3) and to $13.50 \mathrm{~cm} 3 \mathrm{~mol}-1 \mathrm{~K}$ (for 4) at $2.0 \mathrm{~K}$, suggesting a moderately weak antiferromagnetic exchange interaction. In the case of compound 3, fitting of the experimental data was performed by means of Equation (1), by using the PHI computer program.[33] The best-fit parameters obtained were $\mathrm{J}=-0.04(1) \mathrm{cm}-1$ and $\mathrm{g}=2.01(1)$, confirming the existence of antiferromagnetic coupling between the metal centres. The $J$ value is comparable with those found for similar compounds.[12,34]

In complex 5, the values of the $\mathrm{M} \times \mathrm{T}$ product increase slightly, up to a maximum value of $27.39 \mathrm{~cm} 3$ mol-1 $\mathrm{K}$ at $35 \mathrm{~K}$. This increase of $\mathrm{M} \times \mathrm{T}$ might indicate moderate intramolecular ferromagnetic exchange interactions. Below this temperature, the $\mathrm{M} \times \mathrm{T}$ values decrease continuously down to 15.81 $\mathrm{cm} 3 \mathrm{~mol}-1 \mathrm{~K}$ at $2.0 \mathrm{~K}$, which can be attributed mainly to the depopulation of the DyIII mJ sublevels of the ground $\mathrm{J}$ state.

The $\mathrm{M} \times \mathrm{T}$ values for compound 6 continuously decrease when cooling, reaching a plateau at $13 \mathrm{~K}$ and then drops down to $16.26 \mathrm{~cm} 3 \mathrm{~mol}-1 \mathrm{~K}$ at $2 \mathrm{~K}$.

\section{Ac Magnetic Susceptibility Study}

Ac magnetic susceptibility measurements were performed on compounds 1, 4, 5, 6 and 7. Under zero dc magnetic fields, no maxima for the in-phase ( $\left.M^{\prime}\right)$ and/or out-of-phase ( $\left.M^{\prime \prime}\right)$ susceptibility components were observed, probably due to an important quantum tunnelling of the magnetization (QTM) process present in these systems.[35] To suppress the QTM relaxation process, an optimal external dc field of $0.15 \mathrm{~T}$ for $1,0.1 \mathrm{~T}$ for 5 and 6 and $0.2 \mathrm{~T}$ for 7 was applied and slow relaxation of the magnetization was then revealed (Figure 6).[24] In the case of compound 4, even under applied dc fields up to $0.4 \mathrm{~T}$, no frequency or thermal dependencies of $\mathrm{M}^{\prime}$ and/or $\mathrm{M}^{\prime \prime}$ are observed.

Representation of $\mathrm{M}^{\prime \prime}$, measured at different frequencies $(1-1488 \mathrm{~Hz})$ of the $0.4 \times 10-3 \mathrm{~T}$ ac field, at temperatures between 1.8 and $8.0 \mathrm{~K}$, of compound 1 (Figure 6a) shows maxima of the out-of-phase 
component. The ac curves recorded between 1.8 and $3.6 \mathrm{~K}$ have been well-fitted under the generalized Debye model, as is shown in the corresponding Cole-Cole plot (Figure S1). The extracted relaxation parameters are collected in Table S4, showing $\alpha$ values close to zero, which means there is a narrow distribution of the relaxation times and indicates that a single relaxation is mainly involved in the system's relaxation process.[36] Thermal dependency of the relaxation time of the magnetization $(\tau)$ in compound 1 (Figure 6b) reveals that at the highest temperatures, it follows an Orbach relaxation process. The pre-exponential factor $(\tau 0)$ and the effective energy barrier $(\mathrm{Ea})$ between the two ground magnetic states can then be extracted from Arrhenius $\{\tau=\tau 0 \times \exp [\mathrm{Ea} /(\mathrm{kB} \times \mathrm{T})]\}$, giving values of 2.8 $\times 10-6 \mathrm{~s}$ and $10.16 \mathrm{~cm}-1$, respectively, consistent with the values obtained for similar compounds.[37,38] At low temperatures, the rate of $\tau$ derives from linearity, probably due to the presence of other relaxation mechanisms, such as Raman or direct processes.[39] The full temperature range can be fitted with the following model, Equation (2): $\tau-1=\tau 0$

$$
\tau^{-1}=\tau_{0}^{-1} \times \exp \left[-E_{2} /\left(k_{\mathbb{B}} \times T\right]+C \times T^{n}+A \times T\right.
$$

where the first term represents an Orbach process, and the second and third ones represent Raman and direct relaxation processes, respectively. The $\mathrm{n}$ parameter was fixed at 9, the usual value for Kramers ions.[40] The best fit values obtained are: $\tau 0=7.4 \times 10-6 \mathrm{~s}, \mathrm{Ea}=9.5 \mathrm{~cm}-1, \mathrm{C}=0.02 \mathrm{~s}-1 \mathrm{~K}-9$ and $\mathrm{A}=$ $265.21 \mathrm{~s}-1 \mathrm{~K}-1$.

The ac susceptibility measurements were carried out on compound 5 , with a $0.4 \times 10-3 \mathrm{~T}$ ac field oscillating at six frequencies between 10 and $1488 \mathrm{~Hz}$, in the temperature range of 1.8-6.0 K. The outof-phase signal $\mathrm{M}^{\prime \prime}$ versus $v$ and $\mathrm{M}^{\prime \prime}$ versus $\mathrm{T}$ plots are represented in Figure $6 \mathrm{c}$ and Figure S2, respectively, showing the thermal and frequency dependencies of $\mathrm{M}^{\prime \prime}$, but without net maxima in the range of the measured temperatures, which indicates that the QTM is not completely suppressed. Then, the energy barrier and relaxation time cannot be extracted from the Arrhenius equation; therefore, these parameters were obtained using the Debye model and the equation $\ln \left(\mathrm{M}^{\prime \prime \prime} / \mathrm{M}^{\prime}\right)=\ln (\omega \times \tau 0)+\mathrm{Ea} /(\mathrm{kB}$ $\times \mathrm{T}$ ), $[41,35]$ giving, from the best fit [see Figure S9 (right) and Table S5], a value of Ea $=6.6 \mathrm{~cm}-1$ and an exponential factor $\tau 0=8.2 \times 10-7 \mathrm{~s}$, in good agreement with similar DyIII dinuclear complexes.[41] In complexes 6 and 7, frequency-dependent peaks for the $\mathrm{M}^{\prime \prime}$ component can be observed (Figure 6e and g) under a $0.4 \times 10-3 \mathrm{~T}$ ac field oscillating at frequencies between $1-1488 \mathrm{~Hz}$, in the temperature range of 1.8-4.5 K. Frequency dependences of both $\mathrm{M}^{\prime}$ and $\quad \mathrm{M}^{\prime \prime}$ below $3.3 \mathrm{~K}$ for 6 and $3.0 \mathrm{~K}$ for 7 were analyzed, again using the generalized Debye model.[42] The correspondent Cole-Cole plots depicted in Figures S3 and S4 show almost semicircular shapes in all cases, with estimated $\alpha$ values close to zero. The best-fit relaxation parameters are collected in Tables S6 and S7.

The provided relaxation times $(\tau)$ allow the representations of $\ln (\tau)$ versus $T-1$ (Figure $6 \mathrm{f}$ and $\mathrm{h}$ ), showing that at temperatures above $2.7 \mathrm{~K}$ for 6 and $2.2 \mathrm{~K}$ for 7 , the rate of $\tau$ follows the Arrhenius law, giving energy barriers of 7.1 and $5.0 \mathrm{~cm}-1$ for 6 and 7 , respectively, and pre-exponential factors $(\tau 0)$ of 
$2.9 \times 10-6 \mathrm{~s}(6)$ and $9.3 \times 10-6 \mathrm{~s}$ (7). These given values are in good agreement with other similar ErIII and YbIII field-induced SMM compounds found in the literature.[35,37,43] The deviation of the lineal dependence of $\tau$ at low temperatures, for both compounds, suggests the combination of various relaxation processes of the magnetization. Data of compound 6 can be fitted using Equation (3),[44] affording values of $\mathrm{C}=0.08 \mathrm{~s}-1 \mathrm{~K}-9$ and $\mathrm{A}=1296 \mathrm{~s}-1 \mathrm{~K}-1$.

For compound 7, the relaxation times at low temperatures can be modelled considering both Orbach and Raman processes; Equation (4):

$$
\tau^{-1}=\tau_{0}^{-1} \exp \left[-E_{A} /\left(k_{B} \times T\right)\right]+C \times T^{2}
$$

279

The obtained values are $\tau 0=2.2 \times 10-5 \mathrm{~s}, \mathrm{Ea}=3.7 \mathrm{~cm}-1$ and $\mathrm{C}=0.09 \mathrm{~s}-1 \mathrm{~K}-9$.

\section{Photoluminescence Properties}

Excitation and emission spectra of complexes 1,2 and 4-7, recorded in the solid state, at room temperature, are shown in Figure 7 and Figures S6-S9. The excitation spectra show broad bands around $350 \mathrm{~nm}$, corresponding to the $\pi-\pi^{*}$ transitions of the organic ligands in all of the compounds (see Figure S5). Since the GdIII ion has higher excited electronic states that cannot be sensitized with conventional organic ligands, the lu minescence study of compound (3) reveals internal ligand transitions (Figure S7). In the cases of the NdIII, EuIII, TbIII and ErIII compounds, it is possible to observe the $4 \mathrm{f}-4 \mathrm{f}$ metal absorption peaks above $375 \mathrm{~nm}$.

Luminescence measurements of complex 1 (Figure 7) exhibit emissions of the characteristic $\mathrm{f}-\mathrm{f}$ transitions of the NdIII ion in the NIR region. The emission spectra monitored at $\lambda \mathrm{ex}=350 \mathrm{~nm}$ presents a broad band centred at $416 \mathrm{~nm}$, attributed to residual ligand emission in the visible region and the metal $\mathrm{f}-\mathrm{f}$ transitions $4 \mathrm{~F} 3 / 2 \rightarrow 4 \mathrm{I} / 2$ at $902 \mathrm{~nm}$ and $4 \mathrm{~F} 3 / 2 \rightarrow 4 \mathrm{I} 11 / 2$ at $1061 \mathrm{~nm}$ in the NIR region. From the excitation spectra recorded at the most intense NdIII transition 4F3/2 $\rightarrow 4 I 11 / 2(\lambda \mathrm{em}=1061 \mathrm{~nm})$, the highest absorption band corresponds to ligand-centred transitions from $300 \mathrm{~nm}$ to $380 \mathrm{~nm}$. Also, bands from direct NdIII absorption are revealed at $424 \mathrm{~nm}$ for $2 \mathrm{P} 1 / 2 \leftarrow 4 \mathrm{I} / 2 / 2$, at $468 \mathrm{~nm}$ for $2 \mathrm{~K} 15 / 2+4 \mathrm{G} 11 / 2$ $\leftarrow 4 \mathrm{I} 9 / 2$, at $513 \mathrm{~nm}$ for $2 \mathrm{~K} 13 / 2+4 \mathrm{G} 9 / 2 \leftarrow 4 \mathrm{I} / 2 / 2$, at $526 \mathrm{~nm}$ for $4 \mathrm{G} 7 / 2 \leftarrow 4 \mathrm{I} 9 / 2$, at $588 \mathrm{~nm}$ for $2 \mathrm{G} 7 / 2 \leftarrow$ $4 \mathrm{I} 9 / 2$ and at $743 \mathrm{~nm}$ for $4 \mathrm{~F} 7 / 2+4 \mathrm{~S} 3 / 2 \leftarrow 4 \mathrm{I} 9 / 2$ transitions. The intensities ratios between the ligand and metal absorption peaks demonstrate that the NdIII emission in compound 1 is much more efficient when it is excited in the ligand absorption range than at direct metal absorption wavelengths. However, the ligand emission bands present in the emission spectra recorded in the ligand absorption range reveal that the energy transfer is not complete. 
The excitation spectra of compound 2 (Figure S6), recorded by monitoring the 5D0 $\rightarrow$ 7F2 transition of EuIII at $\lambda \mathrm{em}=615 \mathrm{~nm}$, shows broad ligand-centred $\pi^{*} \leftarrow \pi$ transition bands from 316 to $370 \mathrm{~nm}$. At lower energies, it is possible to distinguish the $\mathrm{f}-\mathrm{f}$ transitions of the metal displaying considerably less intensity with respect to the ligand transitions, suggesting that the emission is supported by the antenna effect involving the organic fragment. The emission spectra of 2 (Figure S6), recorded under excitation at $350 \mathrm{~nm}$, results in the characteristic luminescence of $5 \mathrm{D} 0 \rightarrow 7 \mathrm{FJ}(\mathrm{J}=0-4)$ transitions from the EuIII ion. Specific assignments are as follow: 5D0 $\rightarrow$ 7F0 (579 nm), 5D0 $\rightarrow$ 7F1 $(592 \mathrm{~nm})$, DD0 $\rightarrow$ 7F2 (615 $\mathrm{nm}), 5 \mathrm{D} 0 \rightarrow 7 \mathrm{~F} 3(649 \mathrm{~nm})$ and 5D0 $\rightarrow$ 7F4 $(697 \mathrm{~nm})$. The red emission light spectrum is dominated by the hypersensitive 5D0 $\rightarrow$ 7F2 transition, which shows signs of splitting. This fact indicates that the chemical environment around the EuIII ion does not present an inversion centre.[9,45] Moreover, the presence of only one sharp peak corresponding to the 5D0 $\rightarrow$ 7F0 transition reveals that all of the EuIII centres present the same chemical environment, and thus, the same crystal field, which is in good agreement with the X-ray diffraction crystallographic data. The absence of ligand-centred emission bands suggests that the antenna effect in this complex is efficient. In the case of the TbIII compound 4 , the excitation spectrum recorded at $\lambda \mathrm{em}=545 \mathrm{~nm}$, corresponding to the emission band of 5D4 $\rightarrow$ 7F5 transition, shows the ligand absorption bands and weak peaks coming from some $\mathrm{f}-\mathrm{f}$ transitions from the metal (Figure S8). Complex 4 shows the typical TbIII emission spectrum, containing the expected sequence of 5D4 $\rightarrow 7 \mathrm{FJ}(\mathrm{J}=3-6)$ transitions (Figure $\mathrm{S} 8$ ). Specific assignments are as follows: 5D4 $\rightarrow$ 7F6 (489 nm), 5D4 $\rightarrow$ 7F5 (545 nm), 5D4 $\rightarrow$ 7F4 (585 $\mathrm{nm}), 5 \mathrm{D} 4 \rightarrow 7 \mathrm{~F} 3(620 \mathrm{~nm})$ and 5D4 $\rightarrow$ 7F2 $(646 \mathrm{~nm})$. The spectrum is dominated by the 5D4 $\rightarrow$ 7F5 transition, which gives an intense green luminescence output for the solid sample. As for the EuIII complex, the energy transfer between the ligand and the metal in this complex seems to be efficient, due to the absence of ligand emission bands.

The ligand $\pi^{*} \leftarrow \pi$ transition dominates the excitation spectrum of compound 5 (Figure S9) measured at the $4 \mathrm{~F} 9 / 2 \rightarrow 6 \mathrm{H} 13 / 2$ transition $(\lambda \mathrm{em}=572 \mathrm{~nm})$. Then, the antenna effect enhances the $\mathrm{f}-\mathrm{f}$ emission peaks of the DyIII at 478 and $572 \mathrm{~nm}$ under excitation at $350 \mathrm{~nm}$, which are assigned to the $4 \mathrm{~F} 9 / 2 \rightarrow$ $6 \mathrm{HJ}(\mathrm{J}=15 / 2,13 / 2$ transitions, respectively) (Figure S9). The spectrum is dominated by the 4F9/2 $\rightarrow$ $6 \mathrm{H} 15 / 2$ transition, which gives an intense blue luminescence output for the solid sample. Compound 6 shows intense ligand-centred emission at around $416 \mathrm{~nm}$ (Figure 7). In the NIR range, this compound also exhibits the characteristic emission peak corresponding to the transition $4 \mathrm{I} 13 / 2 \rightarrow 4 \mathrm{I} 15 / 2$ at $1531 \mathrm{~nm}$. In the excitation spectra (Figure 7), there are bands corresponding to through-ligand excitation (EL) and others are assigned to through-metal excitation (ELn) at lower energies. The EL/ELn intensity ratio in the NIR emitting complex 6 is lower than those from the EuIII and TbIII complexes. This fact is because the $\mathrm{f}-\mathrm{f}$ transitions for ErIII are less Laporte forbidden, so the direct lanthanide excitation is more significant in this compound.[46]

338 For complex 7, ligand-centred emission is also observed around $416 \mathrm{~nm}$ (Figure 7), and at $993 \mathrm{~nm}$, the 
340 the absorption of the ligand; as the YbIII ion does not present electronic levels in the UV/Vis region, the 341 sensitization of the LnIII emission could be only caused by the ligand antenna effect (Figure 7).[47] 342

343

344 
347 Here, we have presented structural, magnetic and luminescence studies of a family of homo-dinuclear

348 lanthanide compounds based on the use of 2-FBz and phen ligands. We have used a straightforward

349 room-temperature synthetic procedure and we have successfully obtained six new dinuclear lanthanide

350 compounds. From a structural point of view, the diminution of the ionic radius along the $4 \mathrm{f}$ row is

351 translated into two different structures being obtained. Following the same synthetic method; for large

352 ionic radii, compounds 1-6, with the general formula [Ln2(2-FBz)4(NO3)2(phen)2] ( $\mathrm{Ln}=\mathrm{Nd}, \mathrm{Eu}, \mathrm{Gd}$,

$353 \mathrm{~Tb}$, Dy and Er) present nine-coordinate environment metal ions. Meanwhile, in compound [Yb2(2-

354 FBz)6(phen)2] (7), with YbIII having the smallest ionic radius of the series, the coordination number

355 falls to eight, completely changing the ion environment.

356 All of the compounds discussed in this work, except for the GdIII one, display the corresponding $\mathrm{f}-\mathrm{f}$

357 emission luminescence, due to ligand absorption followed by energy transfer to the metal, direct metal

358 absorption or a combination of the two procedures. The EuIII (2), TbIII (4) and DyIII (5) products emit

359 in the visible range, and the NdIII (1), ErIII (6) and YbIII (7) compounds emit in the NIR region.

360 The fitting of the $\mathrm{M} \times \mathrm{T}$ versus T curve of the GdIII (3) compound by the Heisenberg-Dirac-Van

361 Vleck (HDVV) spin Hamiltonian reveals a weak antiferromagnetic interaction between the two LnIII

362 within the dinuclear unit. Additionally, dynamic magnetic measurements reveal field-induced SMM

363 character for compounds 1 and 5-7.

364 Thus, compounds 1, 5, 6 and 7 present both field-induced SMM and luminescence properties and are

365 considered to be multifunctional complexes with potential biomedical applications. 
Starting Materials: $\mathrm{Ln}(\mathrm{NO} 3) 3 \cdot 6 \mathrm{H} 2 \mathrm{O}$ salts, 2-fluorobenzoic acid and 1,10-phenanthroline (Aldrich)

were used as received, without further purification.

371

372

\section{General Syntheses}

Complexes 1-7 were obtained on the basis of a previously proposed synthetic approach.[48] The preparation of all compounds was achieved by the reaction of 2-fluorobenzoic acid (H-2-FBz, $3 \mathrm{mmol})$ and 1,10-phenanthroline (phen, $0.6 \mathrm{mmol})$ dissolved in $\mathrm{EtOH} / \mathrm{H} 2 \mathrm{O}(20 \mathrm{~mL}, \mathrm{v} / \mathrm{v}=50: 50)$ with a solution of the corresponding $\mathrm{Ln}(\mathrm{NO} 3) 3 \cdot 6 \mathrm{H} 2 \mathrm{O}$ salt (0.5 mmol) [ $\mathrm{Ln}=\mathrm{Nd}(1), \mathrm{Eu}(2), \mathrm{Ln}=\mathrm{Gd}(3), \mathrm{Ln}=\mathrm{Tb}(4)$, $\mathrm{Ln}=\mathrm{Dy}(5), \mathrm{Ln}=\mathrm{Er}(6), \mathrm{Ln}=\mathrm{Yb}(7)]$ in $\mathrm{EtOH}(10 \mathrm{~mL})$. The mixture was stirred for $1 \mathrm{~h}$ at room temperature. Good-quality crystals, suitable for X-ray diffraction analysis of compounds $1-7$, were obtained after 5-10 days of slow evaporation.

Compound 1: C52H32F4N6Nd2O14 (1329.32): calcd. C 46.98, H 2.43, N 6.32; found C 46.5, H 2.5, N

6.8. Selected IR bands (KBr pellet): $v^{\sim}=1613$ (vs), 1592 (s), 1395 (vs), 1384 (vs), 1305 (s) cm-1.

Compound 2: C52H32Eu2F4N6O14 (1344.76): calcd. C 46.44, H 2.40, N 6.25; found C 46.8, H 2.4, N

Compound 3: C52H32Gd2F4N6O14 (1355.34): for C 46.08, H 2.38, N 6.20; found C 45.3, H 2.3, N

6.1. Selected IR bands (KBr pellet): $v^{\sim}=1615$ (vs), 1563 (s), 1395 (vs), 1384 (vs), 1306 (s) cm-1.

Compound 4: C52H32F4N6O14Tb2 (1358.67): calcd. C 45.97, H 2.37, N 6.18; found C 46.4, H 2.4, N

6.3. Selected IR bands (KBr pellet): $v^{\sim}=1615$ (vs), 1563 (s), 1399 (vs), 1384 (vs), 1306 (vs) cm-1.

Compound 5: C52H32Dy2F4N6O14 (1365.84): calcd. C 45.73, H 2.36, N 6.15; found C 47.5, H 2.5, N 5.5. Selected IR bands (KBr pellet): $v^{\sim}=1618$ (vs), 1566 (w), 1395 (s), 1384 (vs), 1306 (s) cm-1.

Compound 6: C52H32Er2F4N6O14 (1375.36): calcd. C 45.41, H 2.34, N 6.11; found C 45.6, H 2.5, N

Compound 7: C66H40F6N4O12Yb2 (1541.10): calcd. C 51.44, H 2.62, N 3.63; found C 51.0, H 2.8, N 
were recorded from $\mathrm{KBr}$ pellets with a Perkin-Elmer 380-B spectrophotometer. Solid-state fluorescence spectra were recorded with a Horiba Jobin Yvon SPEX Nanolog fluorescence spectrophotometer at room temperature. Magnetic measurements were performed on solid polycrystalline samples in a

407 Quantum Design MPMS-XL SQUID magnetometer at the Magnetic Measurements Unit of the

408 Universitat de Barcelona. Pascal's constants were used to estimate the diamagnetic corrections, which were subtracted from the experimental susceptibilities to give the corrected molar magnetic susceptibilities.

412 X-ray Diffraction Crystallography: Crystals of 1-3 and 5-7 were mounted in air in a D8VENTURE

413 (Bruker) diffractometer with CMOS detector. The crystallographic data, conditions retained for the 414 intensity data collection and some features of the structural refinements are listed in Table 2 . All of the 415 structures were refined by the least-squares method. Intensities were collected with a multilayer 416 monochromated Mo-K $\alpha$ radiation. Lorentz polarization and absorption corrections were made in all of 417 the samples. The structures were solved by direct methods, using the SHELXS-97 computer 418 program[49] and were refined by the full-matrix least-squares method, using the SHELXL-2014 computer program. [50] The non-hydrogen atoms were located in successive difference Fourier syntheses and were refined with anisotropic thermal parameters on F2. For hydrogen atoms, isotropic CCDC 1822670 (for 1), 1577089 (for 2), 1577090 (for 3), 1577091 (for 5), 1577734 (for 6), and 1577735 (for 7) contain the supplementary crystallographic data for this paper. These data can be obtained free of charge from The Cambridge Crystallographic Data Centre.

426

427

Supporting Information (see footnote on the first page of this article): Tables S1-S7, Figures S1-S9 and crystallographic data in CIF or other electronic format. 


\section{ACKNOWLEDGEMENTS}

432

433 R. V. acknowledges the financial support from the Spanish government Grant CTQ2015-63614-P. S. S

434 also acknowledges the financial support from the Obra Social de la Fundació Bancària La Caixa.

435 
[1] L. Ouahab (Ed.), Multifunctional Molecular Materials, Pan Stanford Publishing Pte Ltd, Singapore, 2013. Stamatatos, Dalton Trans. 2014, 43, 1965-1969. 2014, 43, 11456-11460.

G. Cucinotta, M. Perfetti, J. Luzon, M. Etienne, P.-E. Car, A. Caneschi, G. Calvez, K. Bernot, R. Sessoli, Angew. Chem. Int. Ed. 2012, 51, 1606-1610; Angew. Chem. 2012, 124, 1638.

F. Pointillart, B. Le Guennic, S. Golhen, O. Cador, O. Maury, L. Ouahab, Chem. Commun. 2013, 49, 615-617. 7621.

K. Soussi, J. Jung, F. Pointillart, B. Le Guennic, B. Lefeuvre, S. Golhen, O.Cador, Y. Guyot, O. Maury, L. Ouahab, Inorg. Chem. Front. 2015, 2, 1105-1117.

D. A. Atwood (Ed.), The Rare Earth Elements: Fundamentals and Applications,John Wiley, Chichester (UK), 2005.

[10] L. Li, X. Zhao, N. Xiao, Y. Wang, Z. Wang, S. Yang, X. Zhou, Inorg. Chim. Acta 2015, 426, $107-112$.

[11] B. Chen, Y. Yang, F. Zapata, G. Qian, Y. Luo, J. Zhang, E. B. Lobkovsky, Inorg. Chem. 2006, $45,8882-8886$.

[12] N. C. Anastasiadis, D. A. Kalofolias, A. Philippidis, S. Tzani, C. Raptopoulou, V. Psycharis, C. J. Milios, A. Escuer, S. P. Perlepes, Dalton Trans. 2015, 44, 10200-10209. 2017, 46, 6349-6357. 
[14] D. Gatteschi, R. Sessoli, J. Villain, Molecular Nanomagnets, Oxford University Press, Oxford, 2006.

[15] J. Tang, P. Zhang, Lanthanide Single Molecule Magnets, Springer, Berlin, 2015.

[16] F. S. Guo, B. M. Day, Y. C. Chen, M. L. Tong, A. Mansikkamäki, R. A. Layfield, Angew.

[17] C. A. P. Goodwin, F. Ortu, D. Reta, N. F. Chilton, D. P. Mills, Nature 2017, 548, 439-442.

[18] J. Y. Ge, L. Cui, J. Li, F. Yu, Y. Song, Y. Q. Zhang, J. L. Zuo, M. Kurmoo, Inorg. Chem. 2017, $56,336-343$.

[19] S. Da Jiang, B. W. Wang, H. L. Sun, Z. M. Wang, S. Gao, J. Am. Chem. Soc. 2011, 133, 47304733 .

[20] C. Das, A. Upadhyay, S. Vaidya, S. K. Singh, G. Rajaraman, M. Shanmugam, Chem. Commun. $2015,51,6137-6140$.

[21] J. Jung, F. Le Natur, O. Cador, F. Pointillart, G. Calvez, C. Daiguebonne, O. Guillou, T. Guizouarn, B. Le Guennic, K. Bernot, Chem. Commun. 2014, 50, 13346-13348.

[22] S.-Y. Lin, L. Zhao, H. Ke, Y.-N. Guo, J. Tang, Y. Guo, J. Dou, Dalton Trans. 2012, 41, 3248.

[23] W.-H. Zhu, S. Li, C. Gao, X. Xiong, Y. Zhang, L. Liu, A. K. Powell, S. Gao, Dalton Trans. $2016,45,4614-4621$.

[24] W.-H. Zhu, X. Xiong, C. Gao, S. Li, Y. Zhang, J. Wang, C. Zhang, A. K. Powell, S. Gao, Dalton Trans. 2017, 46, 14114-14121.

[25] L.-L. Luo, X.-L. Qu, Z. Li, X. Li, H.-L. Sun, Dalton Trans. 2018, 47, 925-934.

[26] W. Wen-Hua, L. Du, Y.-R. Zheng, Y.-J. Qu, Z. Kristallogr. 2016, 231, 823-825.

[27] S. Alvarez, P. Alemany, D. Casanova, J. Cirera, M. Llunell, D. Avnir, Coord Chem. Rev. 2005, 249, 1693-1708.

[28] K. P. Carter, S. J. A. Pope, C. L. Cahill, CrystEngComm 2014, 16, 1873-1884.

[29] J. A. Ridenour, K. P. Carter, R. J. Butcher, C. L. Cahill, CrystEngComm 2017, 19, 1172-1189.

[30] S.-J. Sun, D.-H. Zhang, J.-J. Zhang, H.-M. Ye, S.-P. Wang, K.-Z. Wu, J. Mol. Struct. 2010, 977, $17-25$. 
[31] Y.-L. Li, Q.-Y. Liu, C.-M. Liu, Y.-L. Wang, L. Chen, Aust. J. Chem. 2015, 68488.

[32] F. Cimpoesu, F. Dahan, S. Ladeira, M. Ferbinteanu, J. P. Costes, Inorg. Chem. 2012, 51, 11279 11293.

[33] N. F. Chilton, R. P. Anderson, L. D. Turner, A. Soncini, K. S. Murray, J. Comput. Chem. 2013, 34, 1164-1175.

[34] A. Rohde, W. Urland, Inorg. Chim. Acta 2006, 359, 2448-2454.

497

[35] T. Q. Liu, P. F. Yan, F. Luan, Y. X. Li, J. W. Sun, C. Chen, F. Yang, H. Chen, X. Y. Zou, G. M. Li, Inorg. Chem. 2015, 54, 221-228.

[36] Y.-N. Guo, G.-F. Xu, Y. Guo, J. Tang, Dalton Trans. 2011, 40, 9953.

[37] F. Pointillart, O. Cador, B. Le Guennic, L. Ouahab, Coord. Chem. Rev. 2017, 346, 150-175.

[38] A. J. Calahorro, I. Oyarzabal, B. Fernández, J. M. Seco, T. Tian, D. Fairen- Jimenez, E. Colacio, A. Rodríguez-Diéguez, Dalton Trans. 2016, 45, 591-598.

[39] I. F. Díaz-Ortega, J. M. Herrera, T. Gupta, G. Rajaraman, H. Nojiri, E. Colacio, Inorg. Chem. $2017,56,5594-5610$.

[40] J. Long, B. G. Shestakov, D. Liu, L. F. Chibotaru, Y. Guari, A. V. Cherkasov, G. K. Fukin, A. A. Trifonov, J. Larionova, Chem. Commun. 2017, 53, 4706-4709.

[41] A. Mondal, V. Parmar, S. Konar, Magnetochemistry 2016, 2, 35.

[42] S. M. J. Aubin, Z. Sun, L. Pardi, J. Krzystek, K. Folting, L.-C. Brunel, A. L. Rheingold, G. Christou, D. N. Hendrickson, Inorg. Chem. 1999, 38, 5329-5340.

[43] B. H. Koo, K. S. Lim, D. W. Ryu, W. R. Lee, E. K. Koh, C. S. Hong, Chem. Commun. 2012, $48,2519$.

[44] M. V. Marinho, D. O. Reis, W. X. C. Oliveira, L. F. Marques, H. O. Stumpf, M. Déniz, J. Pasán,

] A. de Bettencourt-Dias (Ed.), Luminescence of Lanthanide Ions in Coordination Compounds and Nanomaterials, Wiley-VCH, Weinheim (Germany), 2014. 
516 [46] A. S. Kalyakina, V. V. Utochnikova, I. S. Bushmarinov, I. V. Ananyev, I. L. Eremenko, D. Volz, F. Rönicke, U. Schepers, R. Van Deun, A. L. Trigub, Y. V. Zubavichus, N. P. Kuzmina, S. Bräse, Chem. Eur. J. 2015, 21, 17921-17932.

519 [47] I. Martinić, S. V. Eliseeva, T. N. Nguyen, V. L. Pecoraro, S. Petoud, J. Am. Chem. Soc. 2017, 520 $139,8388-8391$.

521 [48] X. Li, L. Jin, S. Lu, J. Zhang, J. Mol. Struct. 2002, 604, 65-71.

522 [49] G. M. Sheldrick, Acta Crystallogr., Sect. A: Found. Crystallogr. 2008, 64, 112-122.

523 [50] G. M. Sheldrick, Acta Crystallogr., Sect. C: Struct. Chem. 2015, 71, 3-8. 
Scheme 1 Representation of the different coordination modes of the 9-AC ligand.

Figure. 1 (a) Partially labelled plot of compound 2. $\mathrm{H}$ atoms are omitted for clarity. Colour code: grey =

$\mathrm{C}$, blue $=\mathrm{N}$, red $=\mathrm{O}$, yellow $=\mathrm{F}$ and pink $=\mathrm{Eu}$. (b) Coordination polyhedron of the EuIII ions in compound 2 .

Figure.2 Supramolecular arrangement representation in compound 2 on the (111) plane. In blue: $\pi$ stacking interactions; in green: $\mathrm{C}-\mathrm{H} \cdots \mathrm{F}$ intramolecular

535 hydrogen bonds.

536

537

Figure.3. (a) Partially labelled plot of compound 7. $\mathrm{H}$ atoms are omitted for clarity. Colour code: grey = ions in compound 7.

Figure.4 Representation of the supramolecular arrangement of compound 7 on the (111) plane. In blue: $\pi$-stacking interactions; in green: $\mathrm{C}-\mathrm{H} \cdots \mathrm{F}$ hydrogen bonds.

543

544

Figure.5 $\mathrm{M} \times \mathrm{T}$ versus $\mathrm{T}$ plots for compounds $1-7$. The solid blue line corresponds to the best fit for complex 3 (see text).

Figure 6 Representation of the frequency dependence of the out-of-phase component of the ac susceptibility under $0.15 \mathrm{~T} \mathrm{dc}$ field for 1 (a), under $0.1 \mathrm{~T} \mathrm{dc}$ field for 5 (c) and 6 (e) and under a $0.2 \mathrm{~T} \mathrm{dc}$ field for $7(\mathrm{~g})$. Magnetization relaxation time, $\ln (\tau)$ versus $\mathrm{T}-1$ for $1(\mathrm{~b})$, for $6(\mathrm{f})$ and for $7(\mathrm{~h})$. The red and blue lines represent the fitting using the Arrhenius equation and Orbach, Raman and/or direct relaxation processes, respectively (see text).

(d) Representation of the natural logarithm of $\mathrm{M}^{\prime \prime \prime} \quad \mathrm{M}^{\prime}$

Figure.7 Solid-state excitation (red) and emission (black) spectra of compounds 1, 6 and 7 at r. t. 
SCHEME 1
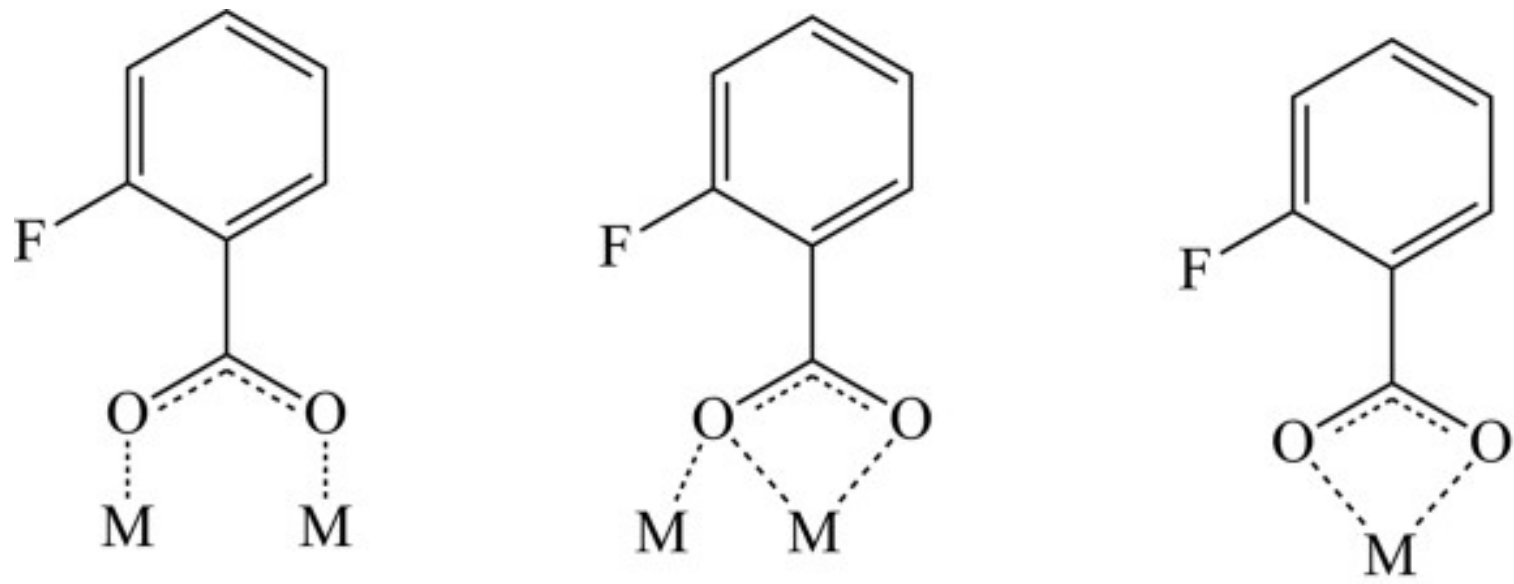

b) chelating-bridging

c) chelating 


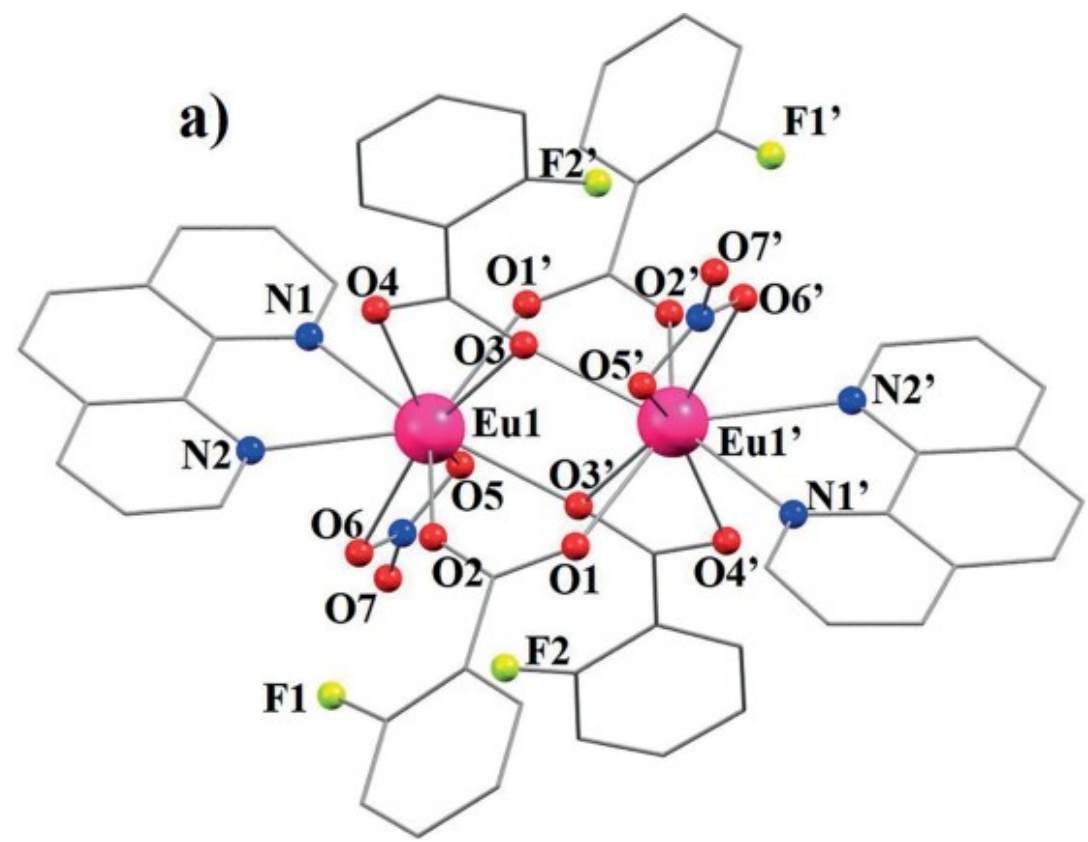

b)

567

568

569 


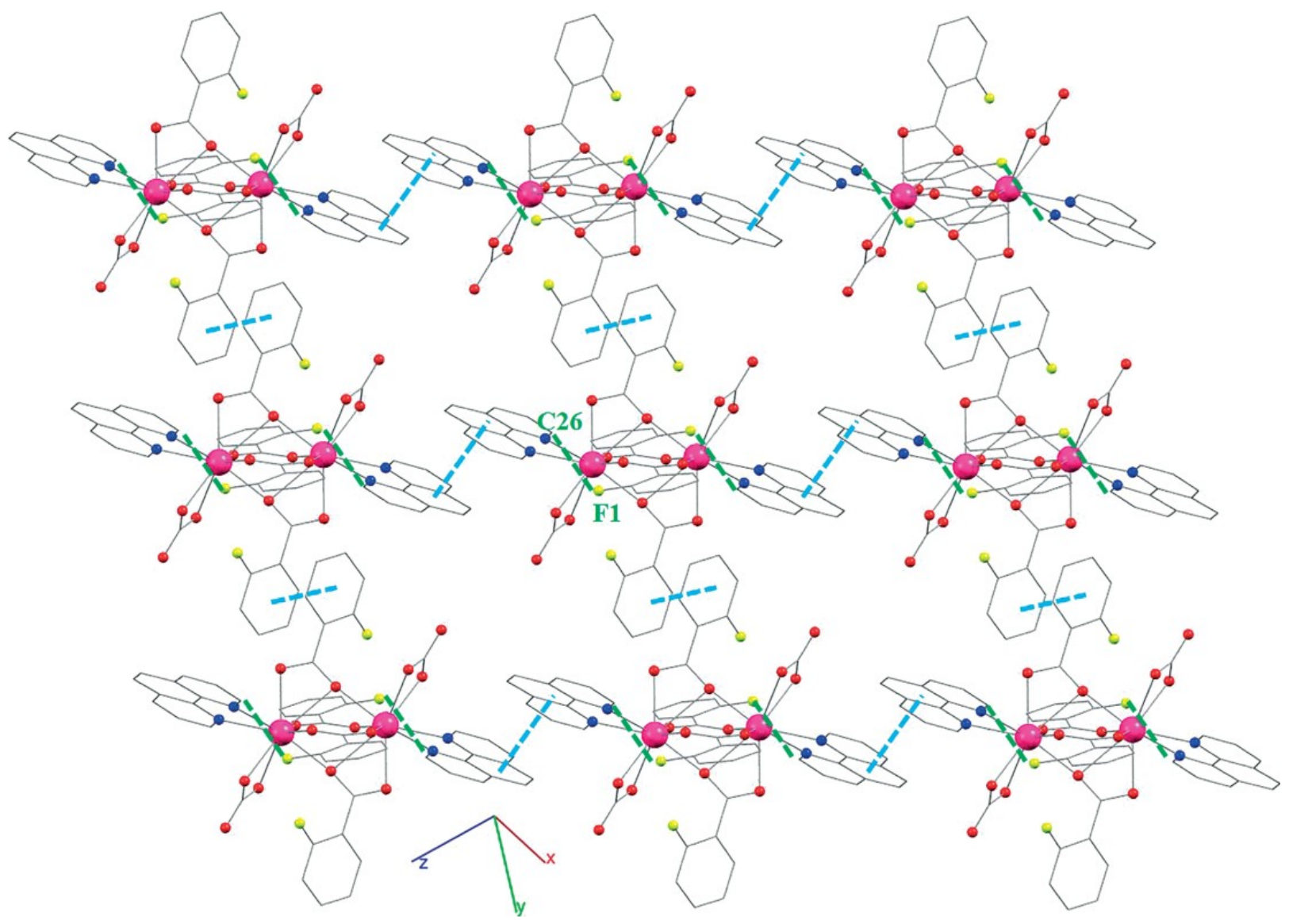

574 

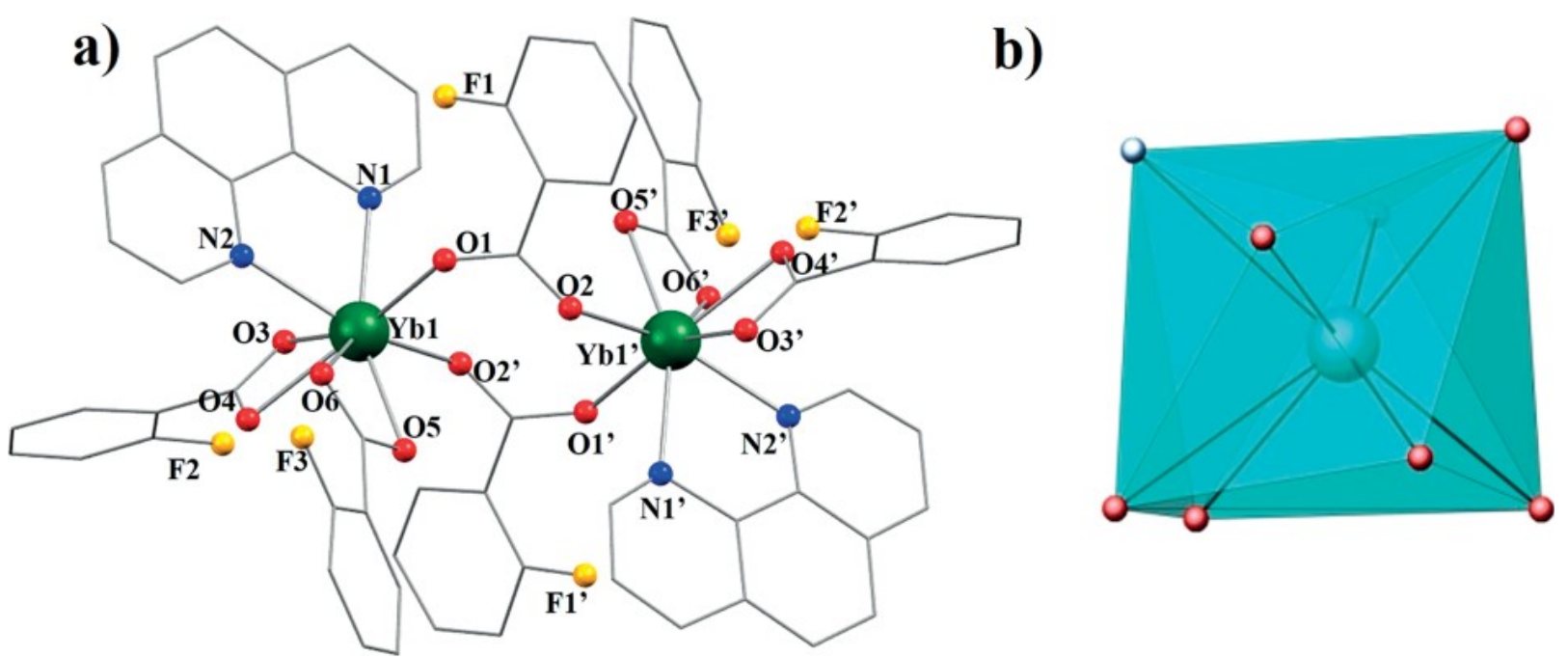
580

581

582

FIGURE 4 


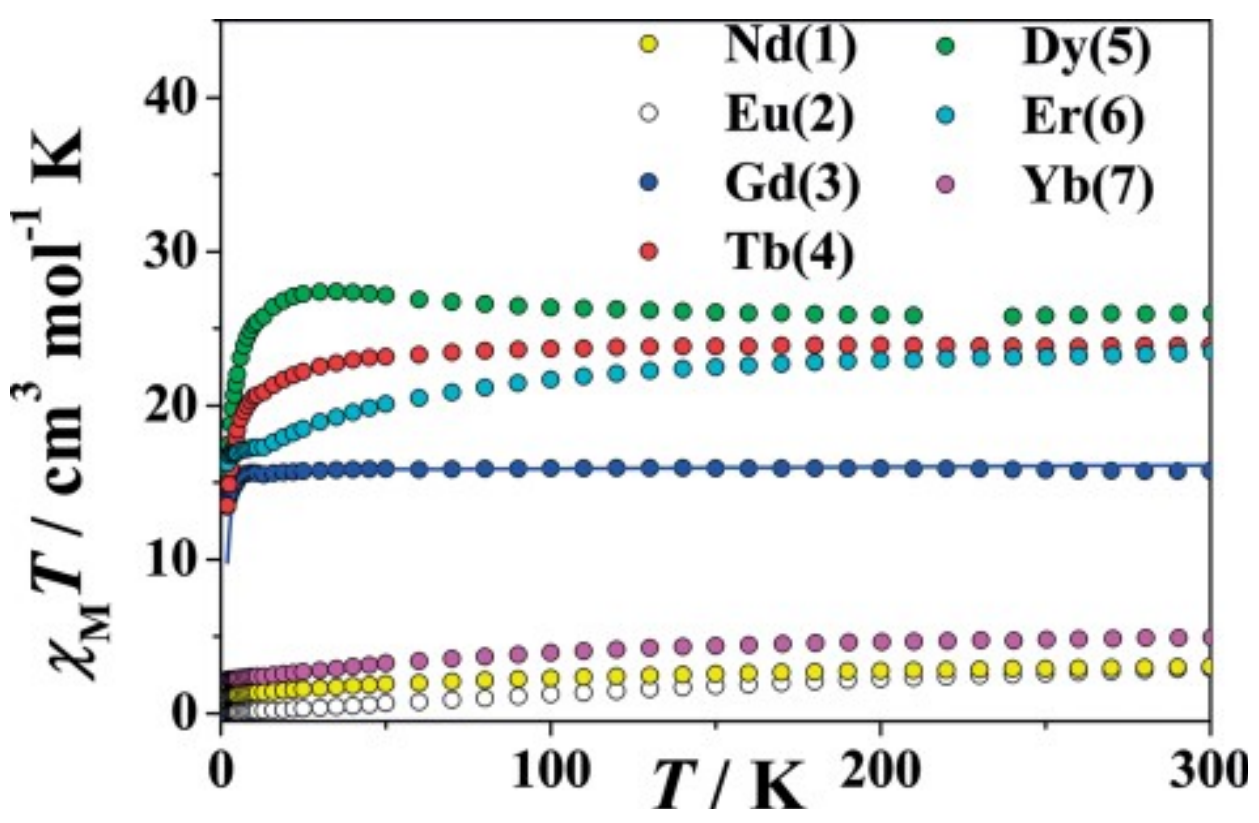



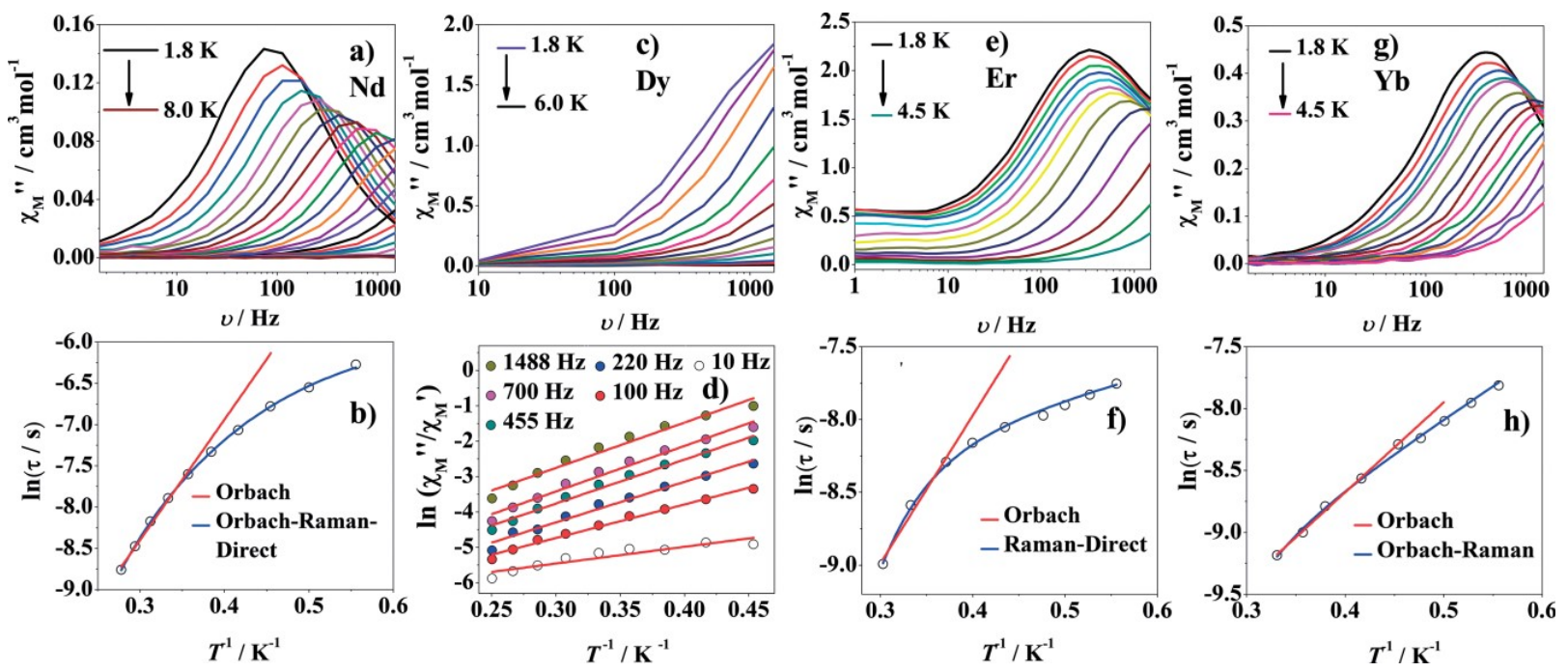

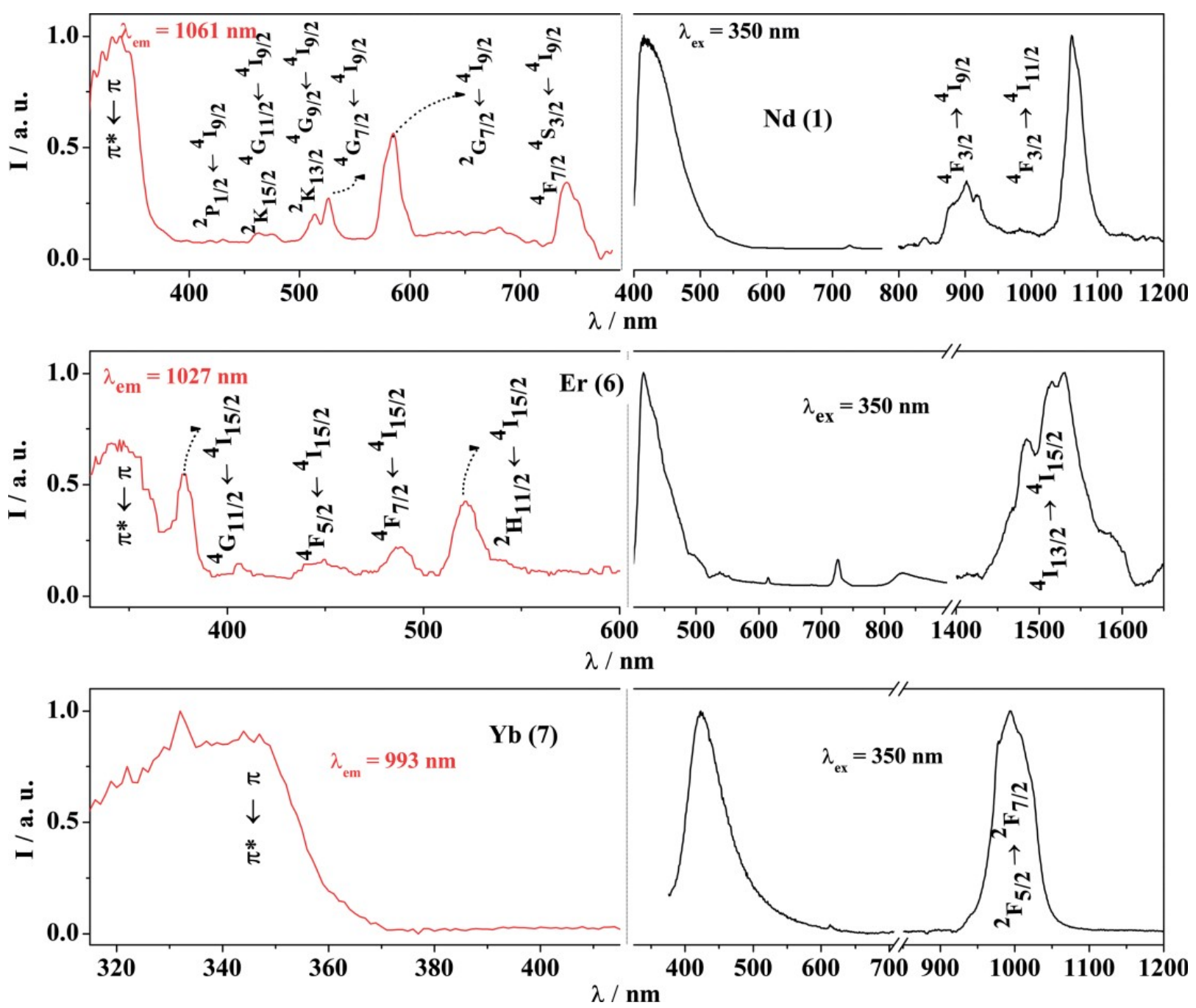
600 Table 1. Selected bond lengths $[\AA]$ for compounds 1-7.

601

\begin{tabular}{|c|c|c|c|c|c|c|c|}
\hline & 1 & 2 & 3 & $4^{20 / 1}$ & 5 & 6 & 7 \\
\hline Ln1-01 & $2.3916(14)$ & $2.3690(1)$ & $23573(1)$ & $2.337(3)$ & $2.3283(2)$ & $23053(12)$ & $2.262(2)$ \\
\hline $\operatorname{Ln} 1-03$ & $24140(15)$ & $2.3705(1)$ & $23588(1)$ & $2340(3)$ & $23265(2)$ & $23008\{13)$ & $2409(2)$ \\
\hline Ln1-04 & $2.4597(15)$ & $2.4066(1)$ & 23932 (1) & $2.374(3)$ & $2.3567(2)$ & $23301(13\rangle$ & $2.376(2)$ \\
\hline Ln1-05 & $2.5671(15)$ & $2.5329(1)$ & 25259:1] & $2.514(4)$ & $2.5056(2)$ & $24923(13)$ & $2.339(3)$ \\
\hline $\operatorname{Ln} 1-06$ & $2.5089(16)$ & $2.4604(1)$ & $24478(1)$ & $2423(4)$ & $24152(2)$ & 23853 (12) & $2.380(3)$ \\
\hline Ln1-N1 & $2.6249(16)$ & $2.5811(1)$ & $25711(1)$ & $2.572(4)$ & $2.5457(2)$ & 25208113) & $2.459(3)$ \\
\hline $\operatorname{Ln} 1-\mathrm{N} 2$ & $26519(17)$ & $2.6170(1)$ & $26094(1)$ & $2.608(5)$ & $2.5862(2)$ & $25659(15)$ & $2.385(6)$ \\
\hline $\operatorname{Ln} 1-02^{\prime}$ & $24151(14)$ & $2.3531(1)$ & $23398(1)$ & $2.327(3)$ & $2.3120(2)$ & $22888(12)$ & $2206(2)$ \\
\hline $\operatorname{Ln} 1-03^{\prime}$ & $2.7052(14)$ & $2.6969(1)$ & $26971(1)$ & $27510)$ & $27326(2)$ & $27432(12)$ & - \\
\hline $\operatorname{Ln} 1 \cdots \operatorname{Ln} 1^{\prime}$ & $4.0015(4)$ & $3.9605(1)$ & $39517(3)$ & $3.9713(8)$ & $3.9497(4)$ & $39294(3)$ & $5.1519(7)$ \\
\hline Symmetry & $1-x, 1-y, 1-z$ & $1-x, 2-y_{0}-z$ & $-x, 2-x, 1-z$ & $-x,-x,-z$ & $1-x_{z} 1-y, 1-z$ & $1-x, 1-y, 1-z$ & $1-x_{v} 1-y, 2-z$ \\
\hline
\end{tabular}

602

603 
604 Table 2 Crystal data and collection details for the X-ray diffraction structure of complexes 1-3 and 5-7.

605

\begin{tabular}{|c|c|c|c|c|c|c|}
\hline & 1 & 2 & 3 & 5 & 6 & 7 \\
\hline \multirow{2}{*}{$\begin{array}{l}\text { Formula } \\
\text { Formula mass [g mol' } \\
\text { ग] }\end{array}$} & \multirow{2}{*}{$\begin{array}{l}\mathrm{C}_{52} \mathrm{H}_{22} \mathrm{~F}_{4} \mathrm{~N}_{6} \mathrm{Nd}_{2} \mathrm{O}_{14} \\
132932\end{array}$} & $\mathrm{C}_{52} \mathrm{H}_{42} \mathrm{Eu}_{2} \mathrm{~F}_{4} \mathrm{~N}_{6} \mathrm{O}_{14}$ & $\mathrm{C}_{52} \mathrm{H}_{22} \mathrm{Gd}_{2} \mathrm{~F}_{4} \mathrm{~N}_{6} \mathrm{O}_{14}$ & $\mathrm{C}_{62} \mathrm{H}_{42} \mathrm{D}_{4} \mathrm{~F}_{4} \mathrm{~N}_{6} \mathrm{O}_{14}$ & $\mathrm{C}_{62} \mathrm{H}_{22} \mathrm{Er}_{2} \mathrm{~F}_{4} \mathrm{~N}_{6} \mathrm{O}_{14}$ & $\mathrm{C}_{62} \mathrm{H}_{40} \mathrm{~F}_{6} \mathrm{~N}_{4} \mathrm{O}_{12} \mathrm{Yb}_{2}$ \\
\hline & & 134476 & 135534 & 1365.84 & 1375.36 & 1541.10 \\
\hline System & triclinic & triclinic & triclinic & triclinic & triclinic & triclinic \\
\hline Space group & PI & PI & PI & pl & PI & PI \\
\hline$a$ [ذ] & $106739(7)$ & $106539(2)$ & $10.6487(3)$ & $106699(7)$ & $10.6433(4)$ & $11.5251(15)$ \\
\hline$b[\dot{\mathrm{k}}]$ & $10.7928(7)$ & $10.8226(3)$ & $10.8322(3)$ & $10.8859(7)$ & $10.8789(4)$ & $11.6743(11)$ \\
\hline$c[$ [A] & $11.2992(7)$ & $11.1880(3)$ & $11.1560(3)$ & $11.1024(7)$ & $11.0468(4)$ & $11.9243(11)$ \\
\hline$a[]$ & $83332(2)$ & $83.521(2)$ & $83.599(2)$ & $83.870(2)$ & $83.902(1)$ & $114.216(8)$ \\
\hline$\beta[]$ & $82070(2)$ & $81.661(2)$ & $81.561(2)$ & $81.282(2)$ & $81.129(1)$ & $99.451(5)$ \\
\hline$y[]$ & $70223(2)$ & $70001(2)$ & $69.942(2)$ & $69.645(2)$ & $69.463(1)$ & $92.998(5)$ \\
\hline$V\left[A^{3}\right]$ & $1209.82(14)$ & $1196.63 / 5]$ & $1193.10(6)$ & $1193.03 / 13)$ & $1181.58(8)$ & $1430.5(3)$ \\
\hline$Z$ & 1 & 1 & 1 & 1 & 1 & 1 \\
\hline$T[\mathrm{~K}]$ & $100[2]$ & $100,2]$ & $100(2)$ & $100(2)$ & $100(2)$ & $100(2)$ \\
\hline $2\left(\mathrm{Mo}_{\mathrm{O}}-\mathrm{K}_{2}\right)[\overline{\mathrm{A}}]$ & 0.71073 & 0.71073 & 0.71073 & Q.71073 & 0.71073 & 0.71073 \\
\hline$D_{\text {eak }}\left[\mathrm{g} \mathrm{cm}^{-2}\right]$ & 1.825 & 1.866 & 1.886 & 1.901 & 1.933 & 1.789 \\
\hline$\mu\left(\mathrm{Mo}_{0}-\mathrm{K}_{2}\right]\left[\mathrm{mm}^{-1}\right]$ & 2.215 & 2.691 & 2.849 & 3.202 & 3.622 & 3.339 \\
\hline $\mathrm{R}$ & $0.0568(7009)$ & $0.0237(6769)$ & 0.0152 (13966) & $0.0254(7176)$ & 0.0151 (6938) & $0.0337(7481)$ \\
\hline$w P_{2}$ & 0.0206 (7260) & $0.0562(7278)$ & $0.0353(15022)$ & $0.0643(7312)$ & $0.0384(7208)$ & 0.0709 (88878) \\
\hline
\end{tabular}

